\title{
Cognitive Cooperation for the Downlink of Frequency Reuse Small Cells
}

\author{
Salam Akoum, ${ }^{1}$ Marie Zwingelstein-Colin, ${ }^{2}$ Robert W. Heath Jr., ${ }^{1}$ and Merouane Debbah ${ }^{3}$ \\ ${ }^{1}$ Wireless Networking and Communications Group, Department of Electrical and Computer Engineering, \\ The University of Texas at Austin, 1 University Station C0803, Austin, TX 78712-0240, USA \\ ${ }^{2}$ IEMN/DOAE, UMR 8520, University Lille Nord de France, 59000 Lille, France \\ ${ }^{3}$ Ecole Superieure d'Electrecite (SUPELEC), Alcatel-Lucent Chair on Flexible Radios, 3 rue Joliot-Curie, \\ 91192 Gif sur Yvette Cedex, France
}

Correspondence should be addressed to Marie Zwingelstein-Colin, marie.zwingelstein-colin@univ-valenciennes.fr

Received 1 June 2010; Revised 28 September 2010; Accepted 16 November 2010

Academic Editor: Robert Schober

Copyright (c) 2011 Salam Akoum et al. This is an open access article distributed under the Creative Commons Attribution License, which permits unrestricted use, distribution, and reproduction in any medium, provided the original work is properly cited.

\begin{abstract}
We develop a cooperative diversity protocol coded over space, time, and frequency to achieve improved quality of service for mobile users in the downlink of small-cell frequency reuse networks. The proposed protocol, called cooperative frequency reuse (CFR), leverages the cellular frequency reuse concept to create space and frequency diversity among pairs of adjacent base stations. The CFR protocol is compatible with the half-duplex mode and is distributed in the sense that each base station acts in autonomy, without the need of a centralized entity. It is implemented in two phases. During the first phase, each base station independently serves its own users on its dedicated frequency band. It simultaneously listens to the symbols transmitted by neighboring base stations. Cognitive cooperation is introduced in the second phase, where each base station transmits on two frequency bands to the scheduled users in both base stations, by means of an appropriately chosen distributed space time code based on the Golden code. We analyze and discuss the performance of the proposed protocol in terms of bit error rate, probability of outage, and ergodic sum rate under different scenarios. Simulation results show that the proposed protocol yields considerable improvement over direct transmission frequency reuse strategies.
\end{abstract}

\section{Introduction}

Small-cell wireless networks provide increased capacity and higher area spectral efficiency [1-3]. The benefits reaped from these networks come, however, at the expense of increased cochannel interference, especially at the cell edge. Conventional cellular networks manage the interference problem by requiring adjacent base stations (BSs) to transmit on different frequency bands. This mechanism is called frequency reuse (FR). It increases the reliability of the cellular networks while at the same time incurring a poor spatial reuse of the expensive frequency spectrum [1]. Fractional frequency reuse (FFR) achieves a higher spatial reuse of the spectrum and is suggested for next generation cellular systems $[4,5]$. It divides the frequency bands into subchannels, to be shared orthogonally among BSs to serve users that are interference limited. It maintains, however, universal frequency reuse in the cell center. Small cell networks encounter conflicting requirements between providing an increased area spectral efficiency and maintaining quality of service for their mobile users. One way to resolve this tradeoff is through combining frequency reuse with cooperation between adjacent BSs. Implementing a cooperation algorithm that leverages the FFR concept of cellular systems achieves the dual benefit of higher reliability and higher spectral efficiency.

Cooperation in cellular networks, depending on the level of data and channel state information (CSI) shared between BSs, can be implemented in several ways [6-9]. Cooperative space diversity $[6,10]$ is one such method. It exploits spatial diversity by implementing a virtual antenna array between adjacent BSs, and distributed space-time codes can be constructed over the formed virtual array to increase the reliability of the system [11-13]. In this paper, we design a 
cooperation protocol based on space and frequency diversity, for cooperation between a pair of adjacent BSs. The proposed protocol, called Cooperative Frequency Reuse (CFR), leverages the frequency reuse concept of cellular systems, creating a virtual multiple-input single-output (MISO) system based on the sharing of OFDM frequency bands among adjacent BSs. It can be applied to cellular systems that use FFR, such as WiMAX and LTE, in a straightforward manner. It is cognitive in the sense that the BSs use, opportunistically in time, the frequency bands allocated to their adjacent BSs to transmit to the mobile users, hence creating cognitive diversity on the downlink of the cellular system.

In contrast to the cooperative multicell transmission strategies available in the literature $[7,14,15]$, where base stations jointly process the downlink signals of the mobile users, thereby creating a multiple-input multiple-output (MIMO) broadcast channel, the proposed CFR protocol implements cooperation through a distributed space time code. It is thus especially suitable for mobile flexible networks [16], where the BSs have limited or no wired backhaul communication. The proposed strategy is different from the cooperative transmit diversity in the multihop relay specification for WiMAX, the IEEE 802.16j standard [17]. In the latter, distributed space time codes are implemented across antennas of the deployed relays and the BS, in the same cell, over the same time and frequency resources. It is also different from the shared relay concept proposed in IEEE $802.16 \mathrm{~m}$ [9], where a relay is placed at the intersection of two or more cells, and used to decode the signals from the intersecting BSs. The CFR protocol also differs from other cooperative protocols proposed in the literature such as in [10-12, 18]. The latter protocols are applied for cooperation among mobile nodes on the uplink of cellular systems and can be used for communication among terminals in adhoc networks.

Assuming a half-duplex mode, whereby nodes cannot transmit and receive at the same time on the same frequency band, the CFR protocol is implemented, for a pair of adjacent BSs, in two phases. During the first phase, each BS serves its own users in a protected band, orthogonal to the frequency bands that the adjacent base stations transmit on. The BS listens, during the same phase, to the signal sent by the cooperating BS on another frequency band. The underlying assumption here is that the wireless link between the pair of adjacent BSs is reliable, which is generally the case in practice, when the BSs have a line of sight channel. In the second phase, the BSs divide their transmit power between two frequency bands: they use one band to serve their own users and the other band for cooperation by relaying the signal of the other BS. During the second phase, the CFR protocol implements a distributed space-time code between the cooperating base stations, based on the Golden code [19]. The Golden code is a full-rate space-time code that achieves the optimal diversity-multiplexing tradeoff (DMT). It has been applied in the cooperative communication literature [20], where it was proven to be optimal for the single-input single-output (SISO) amplify-and-forward (AF) cooperative channel.
In this paper, we establish the benefits of the CFR protocol in terms of bit error rate and probability of outage and achievable ergodic sum rate, through extensive numerical simulations. We first consider the ideal case of an isolated pair of collaborating BSs, where the BS to BS link is assumed perfect. We then extend the analysis to incorporate the imperfections in the BS to BS link, for the isolated twobase station case. We finally consider the effect of other cell interference from neighboring noncooperating base stations. The rest of the paper is organized as follows. We describe the multicell downlink transmission model in Section 2. In Section 3, our CFR protocol is presented and discussed. The performances of the CFR protocol in terms of bit error rate, probability of outage, and ergodic sum rate are evaluated in Section 4.1 for the case of isolated collaborating pair of base stations. Section 4.2 analyses the CFR protocol for the case of an infinite number of adjacent base stations, where each collaborating pair acts as an interferer for the other cooperating pairs in the network. Numerical results are presented in Section 5. Concluding remarks and insights into future work are given in Section 6.

Throughout the paper, the following notation is used. Bold lowercase letters $\mathbf{x}$ are used to denote column vectors, bold uppercase letters $\mathbf{X}$ are used to denote matrices, nonbold letters $x$ are used to denote scalar values, and calligraphic letters $\mathcal{X}$ are used to denote sets or functions of sets. Using this notation, $|x|$ is the magnitude of a scalar, $\|\mathbf{x}\|$ is the vector 2-norm, and $\mathbf{X}^{H}$ is the conjugate transpose of $\mathbf{X}$. We use $\mathbb{E}$ to denote expectation. $\mathbf{I}_{n}$ is the identity matrix of size $n \times n$, and the probability of event $E$ is $\mathbb{P}(E) . i=\sqrt{-1}, \mathbb{Z}$ is the ensemble of relative integers, and $\otimes$ is the Kronecker product for matrices.

\section{Multicell Downlink Transmission Model}

In this section, we present the system model, shown in Figure 1, for the downlink of the multicell network of interest. The BSs and the user terminals are assumed to be equipped with a single antenna. The BSs employ OFDM modulation with $N$ subchannels per OFDM frequency band. They implement fractional frequency reuse, such that the users at the cell edge are protected against intercell interference. In order for the model to be consistent with practical considerations, we assume that the base stations operate in half-duplex mode; that is, they cannot transmit and receive at the same time on the same frequency band. We also assume Time Division Multiple Access (TDMA) as the multiple access scheme. We further consider slow fading, such that the coherence time of the channel is larger than the maximum delay tolerated by the application. We do not account for scheduling, resource allocation, or fairness among users. Only global performance over the OFDM frequency band is considered.

The cellular layout that we consider corresponds to the linear Wyner cellular model [21]. Albeit simple and analytically tractable, this model provides considerable insight into the working of real cellular networks [22-26] in the absence of a more realistic tractable approach to other cell 


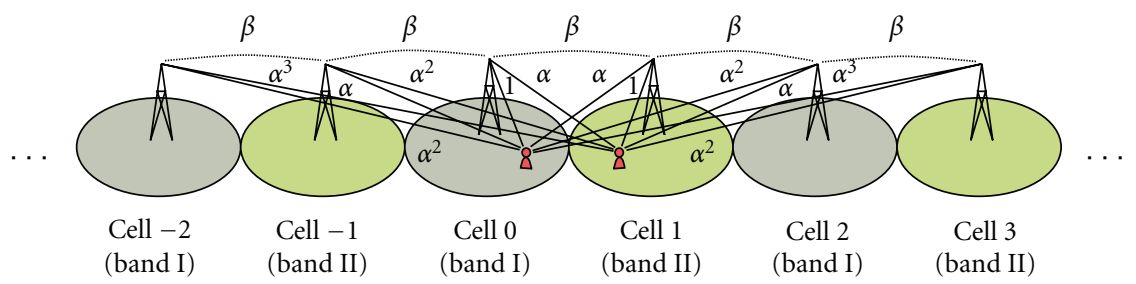

FIGURE 1: Cellular system layout (Wyner model).

interference. It allows for representation of the cellular interference using only one parameter. The linear model is illustrated in Figure 1, where the cells are indexed in increasing order from left to right. The FR factor between adjacent cells is set to 2; that is, 2 OFDM bands, band I and band II, are allocated alternatively to the cells in the linear array. For simplicity, we focus on the cell-edge users, and thus, for fractional frequency reuse, consider only the regions in the cells with frequency reuse greater than 1.

The downlink channel gains account for the effects of path loss as well as Rayleigh fading. The effect of lognormal shadowing is ignored. The path loss is assumed to be invariant to frequency, whereas a different and independent realization of Rayleigh fading is assumed on each OFDM subchannel. In the following, we, respectively, denote by $B_{k}$ and $M_{k}$ the BS and the user terminal in cell $k$. To make the analysis analytically tractable, we set the average channel gain between each base station $B_{k}$ and its user $M_{k}$ to 1 . The average channel gain between $B_{k}$ and a user in an adjacent cell $M_{k+1}$ or $M_{k-1}$ is set to $\alpha$ and that between $B_{k}$ and $M_{k+2}$ or $M_{k-2}$ is set to $\alpha^{2}$, and so on. The link between adjacent base stations is assumed to be an additive white Gaussian noise (AWGN) channel free of fading, with a line of sight component whose gain is denoted by $\beta$.

The signal received by user $k$ at time slot $m$ on OFDM subchannel $n$ can be expressed as

$$
\begin{aligned}
y_{k}(m, n)= & h_{k, k}(m, n) x_{k}(m, n) \\
& +\sum_{l \neq k} h_{\ell, k}(m, n) x_{\ell}(m, n)+w_{k}(m, n),
\end{aligned}
$$

where $x_{k}(m, n)$ is the symbol transmitted by BS $k$ at time slot $m$ and subchannel $n, h_{\ell, k}(m, n)$ is the instantaneous channel gain from BS $\ell$ to user $k$ at time slot $m$ and subchannel $n$, and $w_{k}(m, n)$ is the AWGN with variance $\sigma_{w}^{2}=N_{0}$. The channel gain $h_{\ell, k}(m, n)$ accounts for the effects of Rayleigh fading (independent for each value of $n$ ) and path loss (the same for all $n$ ). Assuming a slow fading scenario, the frame length $T$ is less than the coherence period of the channel (quasistatic assumption); hence, the time index $m$ is, hereafter, omitted in the channel gain notation. We write $h_{\ell, k}(n)$ instead of $h_{\ell, k}(m, n)$. The signal-to-noise ratio is defined as $\mathrm{SNR} \triangleq \mathscr{E} / N_{0}$, where $\mathcal{E}=\mathbb{E}\left\{\left|x_{k}\right|^{2}\right\}$ is the average energy for transmitting a symbol across the link and $N_{0}$ is the variance of the thermal noise observed at the receiver.

The signal received by base station $k$ at time slot $m$ on subcarrier $n$ is

$$
z_{k}(m, n)=\sum_{\ell \neq k} \beta_{\ell, k} x_{\ell}(m, n)+v_{k}(m, n),
$$

where $x_{\ell}(m, n)$ is the symbol transmitted by base station $\ell$ at time slot $m$ and subchannel $n, \beta_{\ell, k}$ is the (deterministic) gain of the link between base station $\ell$ and base station $k$, and $v_{k}(m, n)$ is the AWGN at base station $k$ with variance $\sigma_{v}^{2}$. The channel gain $\beta_{\ell, k}$ is modeled as

$$
\beta_{\ell, k}=\beta^{a+1},
$$

where $a$ is the number of base stations separating base station $\ell$ and base station $k$. In the following, we will consider two cases for the base station to base station link.

(1) Ideal base station to base station link, with $\beta=1$ and $\sigma_{v}^{2}=0$,

(2) nonideal link, with $0 \leq \beta<1$ and $\sigma_{v}^{2}=N_{0}$.

The channel coefficients are assumed to be perfectly known at the receiver, whereas two different scenarios are considered for the channel state information at the transmitter (CSI-T):

(1) no CSI-T,

(2) partial (statistical) CSI-T, where only the statistics of the channel gains are known at the transmitter (the coefficient $\alpha$ ).

\section{Cooperative Frequency Reuse}

In this section, we present the cooperative diversity protocol proposed for improving the quality of service at the cell edge of a small-cell network. The protocol is to be implemented between pairs of adjacent base stations, based on the realistic assumption that the link between two adjacent base stations is, in general, a line-of-sight link that does not manifest severe attenuation.

Assuming fractional frequency reuse and operation in half-duplex mode, each base station transmits information to the scheduled users inside its cell, while at the same time, but on a different frequency band, listens to the signal transmitted by its neighboring base station to the users in the adjacent cell. With some incurred delay, whose effect is neglected in the following analysis, both base stations learn each other's transmitted information and become, thereafter, able to retransmit this information in a collaborative manner, creating a virtual MISO system. Based on this key idea, the proposed protocol is implemented using a distributed spacetime code that optimally exploits the available degrees of freedom.

The cooperative protocol is called Cooperative Frequency Reuse (CFR), as it leverages the frequency reuse cellular concept and allows for cooperation between adjacent base 
stations. Instead of serving the users in their respective cells independently, as is done under classical frequency reuse, the CFR protocol allows neighboring base stations to implement a distributed space-time code to jointly communicate their transmission to their users. The CFR protocol consists of two phases, of duration T/2 each. During the first phase, each base station transmits to its own user on its dedicated OFDM band, and listens to the signal intended to the user of its neighboring base station on the other OFDM band. During the second phase, cooperation is introduced, and each base station transmits to both users on both OFDM bands following the distributed space time code in Table 1.

The CFR protocol can be thought of as a virtual MISO system, where each collaborating BS pair uses a space-time code to increase the reliability of the network. In order not to hinder the achievable rate of the system, we use a fullrate space-time code based on the Golden code [19]. (The Golden code is chosen here because it achieves the diversity multiplexing tradeoff. Other space time codes can be used in the CFR protocol. The Alamouti code for a $2 \times 2 \mathrm{MIMO}$ system can be used for example, at the expense of a loss in the achievable sum rate.) The Golden code is a full-rate, fulldiversity, information lossless and DMT-achieving spacetime code for two transmit and two or more receive antennas. For information symbols $s_{1}, s_{2}, s_{3}$, and $s_{4}$, the Golden code codeword is

$$
\begin{aligned}
\mathbf{X} & =\left[\begin{array}{cc}
\phi\left(s_{1}+\theta s_{2}\right) & \phi\left(s_{3}+\theta s_{4}\right) \\
i \bar{\phi}\left(s_{3}+\bar{\theta} s_{4}\right) & \bar{\phi}\left(s_{1}+\bar{\theta} s_{2}\right)
\end{array}\right] \\
& =\operatorname{diag}\left(\mathbf{M}\left[\begin{array}{l}
s_{1} \\
s_{2}
\end{array}\right]\right)+\operatorname{diag}\left(\mathbf{M}\left[\begin{array}{l}
s_{3} \\
s_{4}
\end{array}\right]\right)\left[\begin{array}{ll}
0 & 1 \\
i & 0
\end{array}\right],
\end{aligned}
$$

where $\mathbf{M}=(1 / \sqrt{5})\left[\frac{\phi}{\phi} \frac{\phi \theta}{\phi \theta}\right]$ is the Golden matrix, $\theta=(1+\sqrt{5}) / 2$ is the golden number, $\bar{\theta}=1-\theta, \phi=1+i(1-\theta)$, and $\bar{\phi}=$ $1+i(1-\bar{\theta})$.

Let the collaborating base station pair and their respective users be denoted by $B_{0}, B_{1}, M_{0}, M_{1}$. The Golden code is adapted to the CFR protocol in the following manner. Prior to transmission, the information symbols $\mathbf{s}=$ $\left[\begin{array}{llll}s_{1} & s_{2} & s_{3} & s_{4}\end{array}\right]$ and $\mathbf{t}=\left[\begin{array}{llll}t_{1} & t_{2} & t_{3} & t_{4}\end{array}\right]$, intended for users $M_{0}$ and $M_{1}$, respectively, are first precoded by the Golden matrix $\mathbf{M}$ as follows:

$$
\begin{aligned}
& {\left[\begin{array}{l}
a_{1} \\
a_{2}
\end{array}\right]=\mathbf{M}\left[\begin{array}{l}
s_{1} \\
s_{2}
\end{array}\right], \quad\left[\begin{array}{l}
a_{3} \\
a_{4}
\end{array}\right]=\mathbf{M}\left[\begin{array}{l}
s_{3} \\
s_{4}
\end{array}\right],} \\
& {\left[\begin{array}{l}
b_{1} \\
b_{2}
\end{array}\right]=\mathbf{M}\left[\begin{array}{l}
t_{1} \\
t_{2}
\end{array}\right], \quad\left[\begin{array}{l}
b_{3} \\
b_{4}
\end{array}\right]=\mathbf{M}\left[\begin{array}{l}
t_{3} \\
t_{4}
\end{array}\right] .}
\end{aligned}
$$

After applying Golden code precoding, the symbols $\left[\begin{array}{llll}a_{1} & a_{2} & a_{3} & a_{4}\end{array}\right]$ and $\left[\begin{array}{llll}b_{1} & b_{2} & b_{3} & b_{4}\end{array}\right]$ are transmitted as shown in Table 2. During the first phase (time slots $2 p$ and $2 p+1)$, base station $B_{0}$ transmits symbols $a_{1}$ and $a_{3}$ to its user on its dedicated OFDM band (band I) and listens to the symbols $b_{1}$ and $b_{3}$ transmitted by base station $B_{1}$ on base station $B_{1}$, dedicated OFDM band (band II). $B_{1}$ transmission of $b_{1}$ and $b_{3}$ follows similarly.
TABLE 1: Proposed CFR protocol, for the pair of base stations numbers 0 and 1, operating on OFDM bands I and II.

\begin{tabular}{rccc}
\hline & 1st phase & 2nd phase \\
\hline \multirow{2}{*}{ Base Station 0 } & Band I & Transmit to user 0 & Transmit to user 1 \\
Band II & Listen to base station 1 & Transmit to user 0 \\
\hline Base Station 1 Band I & Listen to base station 0 & Transmit to user 1 \\
Band II & Transmit to user 1 & Transmit to user 0 \\
\hline
\end{tabular}

During the second phase (time slots $2 p+1$ and $2 p+2$ ), a space time Golden code codeword is transmitted to each user through cooperation between the two base stations, that is, the Golden code codeword $\mathbf{X}_{0}=\left[\begin{array}{cc}a_{1} & a_{3} \\ i a_{4} & a_{2}\end{array}\right]$ is transmitted to user $M_{0}$ on band II, and the Golden code codeword $\mathbf{X}_{1}=$ $\left[\begin{array}{ll}b_{1} & b_{3} \\ i b_{4} & b_{2}\end{array}\right]$ is transmitted to user $M_{1}$ on band $\mathrm{I}$.

Amplify and Forward (AF) is chosen as the relaying protocol, as depicted in Table 2. The amplifying parameter $b$ is used to satisfy the energy constraint

$$
\mathbb{E}\left\{\left|b z_{k}\right|^{2}\right\} \leq \mathcal{E}, \quad \text { that is, } \quad b \leq \sqrt{\frac{\mathscr{E}}{\beta^{2} \mathcal{E}+N_{0}}} .
$$

The coefficients $\gamma_{0}, \bar{\gamma}_{0}=1-\gamma_{0}, \gamma_{1}$, and $\bar{\gamma}_{1}=1-\gamma_{1}$ account for the power control. In case of no CSI-T, they are set equal to $1 / 2$ to reflect the fact that power is equally allocated to the two OFDM bands. In case of partial (statistical) CSI-T at the transmitter, they can be optimized based on the channel gains statistics.

The distributed space-time code is expected to provide a diversity order of 3 (observe from Table 2 that each symbol is transmitted through 3 channel realizations, due to spatial and frequency diversity). Furthermore, in the event of failure of the cooperating links, the Golden code codewords $\mathbf{X}_{0}$ and $\mathbf{X}_{1}$ are still transmitted to users 0 and 1 , but as frequency time codewords (over the 4 time slots of the CFR protocol), instead of space frequency time codewords (implemented over the last 2 time slots over the CFR protocol). Consequently, in case of cooperation failure, the diversity order is reduced to 2 , but the benefits of using the Golden code are preserved. The analysis in Sections 4.1 and 4.2 discusses the performance of the CFR protocol in terms of bit error rate, probability of outage, and average achievable sum rate and compares the CFR with the classical, noncooperative, frequency reuse transmission scheme.

\section{Performance Analysis}

In this section, we derive expressions for the performance evaluation of the CFR protocol based on three metrics, the bit error rate, the probability of outage, and the average achievable sum rate. We first consider the CFR protocol for the ideal case of two isolated adjacent base stations, we then extend the analysis to include other cell interference from noncooperating adjacent base stations, employing the Wyner linear cellular model. 
TABLE 2: Proposed CFR protocol based on the distributed Golden code.

\begin{tabular}{lccccc}
\hline \multicolumn{5}{c}{} & \multicolumn{2}{c}{ 1st phase } & \multicolumn{2}{c}{ 2nd phase } \\
\hline \multirow{3}{*}{ Base station $A$} & $m=2 p$ & $m=2 p+1$ & $m=2 p+2$ & $m=2 p+3$ \\
& Band I $(n)$ & $\mathbf{a}_{\mathbf{1}}$ & $\mathbf{a}_{3}$ & $\sqrt{\bar{\gamma}_{0}} b z_{0}(2 p, n+N)$ & $\sqrt{\bar{\gamma}_{0}} b z_{0}(2 p+1, n+N)$ \\
& Band II $(n+N)$ & $\mathbf{z}_{0}(\mathbf{2} \mathbf{p}, \mathbf{n}+\mathbf{N})$ & $\mathbf{z}_{0}(\mathbf{2} \mathbf{p}+\mathbf{1}, \mathbf{n}+\mathbf{N})$ & $\sqrt{\gamma_{0}} i a_{4}$ & $\sqrt{\gamma_{0}} a_{2}$ \\
\hline \multirow{2}{*}{ Base station $B$} & Band I $(n)$ & $\mathbf{z}_{\mathbf{1}}(\mathbf{2} \mathbf{p}, \mathbf{n})$ & $\mathbf{z}_{\mathbf{1}}(\mathbf{2} \mathbf{p}+\mathbf{1}, \mathbf{n})$ & $\sqrt{\gamma_{1}} i b_{4}$ & $\sqrt{\gamma_{1}} b_{2}$ \\
& Band II $(n+N)$ & $\mathbf{b}_{\mathbf{1}}$ & $\mathbf{b}_{\mathbf{3}}$ & $\sqrt{\gamma_{1}} b z_{1}(2 p, n)$ & $\sqrt{\gamma_{1}} b z_{1}(2 p+1, n)$ \\
\hline
\end{tabular}

Light-faced font: transmitted

Bold font: received.

For the classical noncooperative (NC-FR) scheme, the signal received by user $M_{k}$ in time slot $m$ on subchannel $n$ is written as

$$
y_{k}^{\mathrm{NC}-\mathrm{FR}}(m, n)=h_{k, k}(n) x_{k}(m, n)+w_{k}(m, n), \quad k=0,1 .
$$

The instantaneous capacity of user $M_{k}$ on subchannel $n$ assuming Gaussian transmitted signals thus follows as

$$
C_{k}^{\mathrm{NC}-\mathrm{FR}}=\log _{2}\left(1+\frac{\mathcal{E}}{N_{0}}\left|h_{k, k}(n)\right|^{2}\right) \mathrm{bpcu},
$$

where bpcu is the capacity unit in bits per channel use.

The joint probability of outage for users $M_{0}$ and $M_{1}$, served by BSs $B_{0}$ and $B_{1}$, respectively, is

$$
P_{O}^{\mathrm{NC}-\mathrm{FR}}(\mathrm{R})=\mathbb{P}\left(C_{0}^{\mathrm{NC}-\mathrm{FR}}<\mathrm{R}, C_{1}^{\mathrm{NC}-\mathrm{FR}}<\mathrm{R}\right),
$$

where $R$ is the target spectral efficiency of the system, and the ergodic sum rate is further given by

$$
\begin{aligned}
\mathrm{R}^{\mathrm{NC}-\mathrm{FR}} & =\mathbb{E}\left\{C_{0}^{\mathrm{NC}-\mathrm{FR}}+C_{1}^{\mathrm{NC}-\mathrm{FR}}\right\} \\
& =\mathbb{E}\left\{C_{0}^{\mathrm{NC}-\mathrm{FR}}\right\}+\mathbb{E}\left\{C_{1}^{\mathrm{NC}-\mathrm{FR}}\right\} .
\end{aligned}
$$

To decode $x_{k}$ under the assumption of equally likely transmitted constellation points, the maximum likelihood (ML) receiver for the classical scheme is given by

$$
\hat{x}_{k}^{\mathrm{NC}-\mathrm{FR}}=\arg \min _{x_{k} \in \mathcal{N}^{\mathrm{NC}-\mathrm{FR}}}\left[\left(y_{k}^{\mathrm{NC}-\mathrm{FR}}-h_{k, k} x_{k}\right)^{*}\left(y_{k}^{\mathrm{NC}-\mathrm{FR}}-h_{k, k} x_{k}\right)\right],
$$

where $\mathcal{N}^{\mathrm{NC}-\mathrm{FR}}$ is the set of all possible transmitted symbols. The bit error rate (BER) under ML decoding is computed from the probability of symbol error, depending on the constellation used for symbol mapping at the base station.

4.1. The CFR Protocol for Two Isolated Base Stations. In this section, we characterize the performance of the CFR protocol presented in Section 3 in comparison with the classical, non cooperative, FR scheme, in the ideal case of $N=2$ isolated base stations. We assume that the cells outside the collaborating pair of interest are not a source of interference.

For the CFR protocol, the received signal at user $M_{k}$ is written, in vector form, as

$$
\mathbf{y}_{k}^{\mathrm{CFR}}=\mathbf{H}_{k} \mathbf{x}_{k}+\mathbf{v}_{k}+\mathbf{w}_{k}, \quad k=0,1
$$

For the user terminal $M_{0}$, it follows from Section 3, as shown in Table 2, that

$$
\begin{aligned}
\mathbf{y}_{0}^{\mathrm{CFR}}=\left[y_{0}(2 p, n), y_{0}(2 p+1, n),\right. \\
\left.\quad y_{0}(2 p+2, n+N), y_{0}(2 p+3, n+N)\right]^{T},
\end{aligned}
$$

where the transmitted signal vector $\mathbf{x}_{0}$ is

$$
\mathbf{x}_{0}=\left[\begin{array}{llll}
a_{1} & a_{3} & i a_{4} & a_{2}
\end{array}\right]^{T} .
$$

The channel matrix $\mathbf{H}_{0}$ is written as

$$
\mathbf{H}_{0}=\left[\begin{array}{cc}
h_{0,0}(n) & 0 \\
\sqrt{\bar{\gamma}_{1}} h_{1,0}(n+N) b \beta & \sqrt{\gamma_{0}} h_{0,0}(n+N)
\end{array}\right] \otimes\left[\begin{array}{ll}
1 & 0 \\
0 & 1
\end{array}\right],
$$

and the noise components, $\mathbf{v}_{0}$, received during the first phase and amplified by the AF relaying protocol in the second phase are given by

$$
\mathbf{v}_{0}=\left[\begin{array}{c}
0 \\
0 \\
\sqrt{\bar{\gamma}_{1}} h_{1,0}(n+N) b v_{1}(2 p, n) \\
\sqrt{\bar{\gamma}_{1}} h_{1,0}(n+N) b v_{1}(2 p+1, n)
\end{array}\right]
$$

The AWGN noise vector is finally written as

$$
\mathbf{w}_{0}=\left[\begin{array}{c}
w_{0}(2 p, n) \\
w_{0}(2 p+1, n) \\
w_{0}(2 p+2, n+N) \\
w_{0}(2 p+3, n+N)
\end{array}\right]
$$

Similarly, for user terminal $M_{1}$, in cell 1 , the received signal vector $\mathbf{y}_{1}^{C F R}$ is given by

$$
\begin{gathered}
\mathbf{y}_{1}^{\mathrm{CFR}}=\left[y_{1}(2 p, n+N), y_{1}(2 p+1, n+N),\right. \\
\left.y_{1}(2 p+2, n), y_{1}(2 p+3, n)\right]^{T},
\end{gathered}
$$


where

$$
\begin{aligned}
& \mathbf{x}_{1}=\left[\begin{array}{llll}
b_{1} & b_{3} & i b_{4} & b_{2}
\end{array}\right]^{T}, \\
& \mathbf{H}_{1}=\left[\begin{array}{cc}
h_{1,1}(n+N) & 0 \\
\sqrt{\bar{\gamma}_{0}} h_{0,1}(n) b \beta & \sqrt{\gamma_{1}} h_{1,1}(n)
\end{array}\right] \otimes\left[\begin{array}{ll}
1 & 0 \\
0 & 1
\end{array}\right], \\
& \mathbf{v}_{1}=\left[\begin{array}{c}
0 \\
0 \\
\sqrt{\bar{\gamma}_{0}} h_{0,1}(n) b v_{0}(2 p, n+N) \\
\sqrt{\bar{\gamma}_{0}} h_{0,1}(n) b v_{0}(2 p+1, n+N)
\end{array}\right] \text {, } \\
& \mathbf{w}_{1}=\left[\begin{array}{c}
w_{1}(2 p, n+N) \\
w_{1}(2 p+1, n+N) \\
w_{1}(2 p+2, n) \\
w_{1}(2 p+3, n)
\end{array}\right] .
\end{aligned}
$$

The instantaneous capacity of user $M_{k}$ under the CFR protocol follows as

$$
\begin{aligned}
C_{k}^{\mathrm{CFR}}=\frac{1}{4} \log _{2}(\operatorname{det} & \left(\mathbf{I}_{4}+\mathbf{H}_{k} \mathbb{E}\left\{\mathbf{x}_{k} \mathbf{x}_{k}^{H}\right\} \mathbf{H}_{k}^{H} \mathbb{E}^{-1}\right. \\
& \left.\left.\times\left\{\left(\mathbf{v}_{k}+\mathbf{w}_{k}\right)\left(\mathbf{v}_{k}+\mathbf{w}_{k}\right)^{H}\right\}\right)\right) \text { bpcu, } k=0,1 .
\end{aligned}
$$

The joint probability of outage for users $M_{0}$ and $M_{1}$ is

$$
P_{\mathrm{O}}^{\mathrm{CFR}}(\mathrm{R})=\mathbb{P}\left(C_{0}^{\mathrm{CFR}}<\mathrm{R}, C_{1}^{\mathrm{CFR}}<\mathrm{R}\right),
$$

where $\mathrm{R}$ is the target spectral efficiency. The average sum rate is given by

$$
\mathrm{R}^{\mathrm{CFR}}=\mathbb{E}\left\{C_{0}^{\mathrm{CFR}}+C_{1}^{\mathrm{CFR}}\right\}
$$

To analyse the bit error rate of the CFR protocol, ML decoding is implemented

$$
\widehat{\mathbf{x}}_{k}^{\mathrm{CFR}}=\arg \min _{\mathbf{x}_{k} \in \mathcal{N}}\left[\left(\mathbf{y}_{k}^{\mathrm{CFR}}-\mathbf{H}_{k} \mathbf{x}_{k}\right)^{*}\left(\mathbf{y}_{k}^{\mathrm{CFR}}-\mathbf{H}_{k} \mathbf{x}_{k}\right)\right],
$$

where $\mathcal{N}$ is the set of all possible transmitted Golden code codewords.

To analyze the performance of the CFR protocol in terms of probability of outage, ergodic sum rate, and bit error rate, we numerically evaluate the expressions in (21), (22), and (23), respectively, for the QAM constellation of interest. We compare them to the results obtained from the classical NC-FR protocol in (9), (10), and (11). To account for the effect of CSI-T at the base stations, we analyze the performance of the CFR algorithm with and without channel state information at the transmitter. When channel state information is available at the transmitter, the fractional power control factors $\gamma_{k}$ are adjusted to optimize the performance metric in question. For instance, the problem of minimizing the joint probability of outage, for $M_{0}$ and $M_{1}$, is written as follows.
Problem 1 . Find the optimal $\hat{\gamma}_{0}, \hat{\gamma}_{1}$ such that the probability that both users are in outage $\mathbb{P}\left(C_{0}^{C F R}<R, C_{1}^{C F R}<R\right)$ is minimized. In other words,

$$
\begin{aligned}
\left(\hat{\gamma}_{0}, \hat{\gamma}_{1}\right) & =\underset{\gamma_{0}, \gamma_{1}}{\arg \min }\left(C_{0}^{\mathrm{CFR}}<\mathrm{R}, C_{1}^{\mathrm{CFR}}<\mathrm{R}\right) \\
& =\underset{\gamma_{0}, \gamma_{1}}{\arg \min }\left(\mathbb{P}\left(C_{0}^{\mathrm{CFR}}<\mathrm{R}\right) \mathbb{P}\left(C_{1}^{\mathrm{CFR}}<\mathrm{R}\right)\right) .
\end{aligned}
$$

Finding the fractional power allocations that minimize the joint probability of outage requires finding the probability distribution of the instantaneous capacities $C_{0}^{\mathrm{CFR}}$ and $C_{1}^{\mathrm{CFR}}$ in terms of $\gamma_{k}$ and $\gamma_{(k+1)}$. When channel state information is not available at the transmitter, the fractional power factors are equally allocated between the two frequency bands $\gamma_{0}=$ $\gamma_{1}=1 / 2$. Unfortunately, closed-form expressions for these optimization problems cannot be evaluated for finite signalto-noise ratio levels.

For the link between the base stations, we consider, as discussed in Section 3, both the ideal case and the nonideal case with the line-of-sight channel with AWGN noise. Numerical results in Section 5 show the performance of the CFR protocol for all the cases of interest, using Monte Carlo simulations.

4.2. The CFR Protocol for $N$ Base Stations. We extend the analysis in Section 4.1 to the more realistic case of a network consisting of $N>2$ base stations. For this scenario, the base stations still collaborate on a pair basis, but the interference from the noncooperating pair on the user terminals is taken into account. The collaborating pairs are assumed static, in the sense that if base station $B_{k}$ collaborates with adjacent base station $B_{k+1}$, the collaborating pair remains fixed throughout the duration of the cooperative protocol and does not change based on received signal strength at the user terminals. For simplicity, we consider the performance of the base station pairs $\left(B_{k}, B_{k+1}\right)=\left(B_{0}, B_{1}\right)$ with interference from the pairs ..., $\left(B_{-4}, B_{-3}\right),\left(B_{-2}, B_{-1}\right),\left(B_{2}, B_{3}\right),\left(B_{4}, B_{5}\right) \ldots$ (see Figure 1).

The received signal at user $M_{k}$, in cell $B_{k}$, is written in vector form as

$$
\mathbf{y}_{k}^{\mathrm{CFR}, N}=\mathbf{H}_{k} \mathbf{x}_{k}+\mathbf{u}_{k}+\mathbf{v}_{k}+\mathbf{w}_{k}, \quad k=0,1,
$$

where $\mathbf{x}_{k}, \mathbf{H}_{k}, \mathbf{v}_{k}$, and $\mathbf{w}_{k}$ are given by (14), (15), (16), and (17), respectively, and

$$
\mathbf{u}_{k}=\left[\begin{array}{c}
\sum_{\substack{i \in \mathbb{Z} \\
i \neq k}} h_{2 i-k, k}(n+k N) x_{2 i-k}(2 p, n+k N) \\
\sum_{\substack{i \in \mathbb{Z} \\
i \neq k}} h_{2 i-k, k}(n+k N) x_{2 i-k}(2 p+1, n+k N) \\
\sum_{\substack{i \in \mathbb{Z} \\
i \neq 0 \\
i \neq 1}} h_{i, k}(n+(1-k) N) x_{i}(2 p+2, n+(1-k) N) \\
\sum_{\substack{i \in \mathbb{Z} \\
i \neq 0 \\
i \neq 1}} h_{i, k}(n+(1-k) N) x_{i}(2 p+3, n+(1-k) N) \\
k=0,1
\end{array}\right],
$$


corresponds to the vector of interfering signals from the base stations outside the collaborating pair.

The performance of the CFR protocol for the linear Wyner model with $N$ base stations is evaluated, similarly to Section 4.1, based on Monte Carlo simulations in Section 5. Expressions for the performance metrics such as bit error rate, joint probability of outage, and ergodic sum rate are derived using $\mathrm{y}_{k}^{\mathrm{CFR}, N}$, and the effect of the additional interference term $\mathbf{u}_{k}$ is evaluated for the ideal and the nonideal CSI-T cases of interest.

\section{Numerical Results and Discussion}

We provide numerical results to evaluate the performance of the proposed cooperative frequency reuse algorithm in terms of achievable bit error rate, probability of outage, and ergodic sum rate. For our simulations, we consider a linear Wyner model cellular setup, where all cells have the same radius and users are uniformly distributed inside each cell, assuming one sector per cell.

When partial (statistical) CSI-T is available at the transmitter, the power factors $\gamma_{0}$ and $\gamma_{1}$ are chosen from the discrete set $\left(\gamma_{0}, \gamma_{1}\right) \in\{0,0.25,0.5,0.75,1\}$ in order to optimize the performance metric in question (i.e., the bit error rate, the probability of outage, or the ergodic sum rate). For the case of no CSI-T, the power factors $\gamma_{0}=\gamma_{1}=1 / 2$ are allocated equally between the two frequency bands.

We start by examining the performance of the CFR protocol for the case of two isolated base stations. We present in Figures 2, 3, and 4, respectively, the bit error rate, the probability of outage, and the average sum rate as a function of the signal-to-noise ratio, for the CFR protocol with the following scenarios:

(1) no CSI-T, ideal link between the base stations ( $\beta=1$ and $\sigma_{v}^{2}=0$ ),

(2) no CSI-T, non ideal (worst case) link quality between the base stations $\left(\beta=\alpha\right.$ and $\left.\sigma_{v}^{2}=N_{0}\right)$,

(3) CSI-T, ideal link between the base stations,

(4) CSI-T, non ideal (worst case) link quality between the base stations.

Figure 2 plots the bit error rate versus SNR, for symbols $s$ and $t$ chosen from a 4-QAM and a 16-QAM constellation. The link quality between the adjacent base stations and the user terminal is set equal to $\alpha=0.1$. For comparison, we include in the figure the bit error rate for the classical frequency reuse FR 2 scheme, as well as the bit error rate for the universal frequency reuse, FR 1 scheme, where the latter's symbols are taken from a BPSK and a 4-QAM constellation, respectively, for fairness of comparison. It can be observed from Figure 2 that even in the absence of CSI$\mathrm{T}$, for the worst case of nonideal link, the CFR protocol outperforms the classical FR 2 protocol, for all signal-tonoise ratio values. The FR 1 scheme outperforms the CFR protocol at low SNR, where the distributed space-time code does not perform well. At high SNR values, the diversity obtained from the CFR protocol outperforms that of the

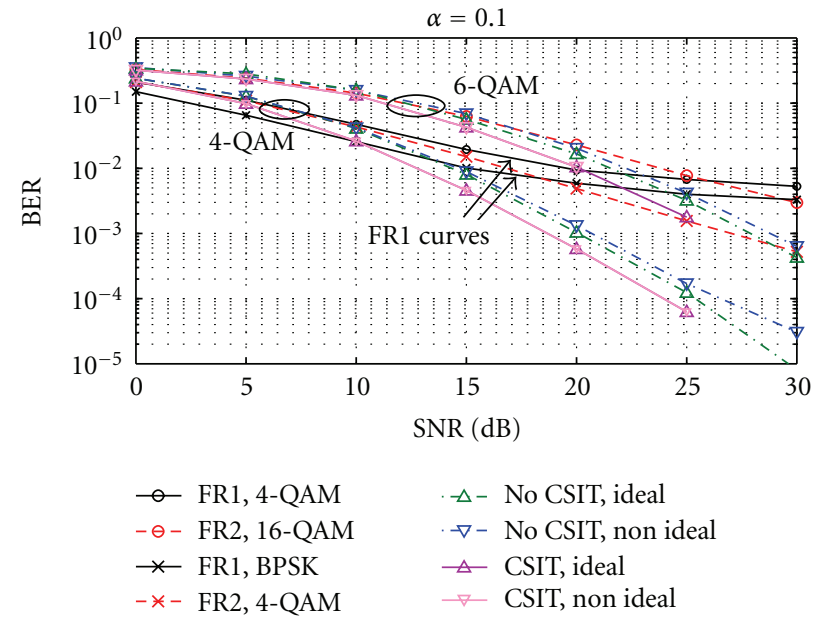

FIGURE 2: Bit error rate (BER), as a function of the SNR (dB), for two isolated base stations. The link quality $\alpha=0.1$, and the modulation is 4-QAM and 16-QAM, respectively. The FR1 curves denote the direct transmission scheme with BPSK and 4-QAM modulation, respectively. The non ideal link denotes the case when the base station-to-base station link gain is $\beta=\alpha$.

FR 1 direct transmission protocol, and the CFR protocol achieves the best performance. The good performance of the CFR protocol at high SNR can be well invested for application in the small-cell scenario. Due to the proximity of the base station from its intended receiver in a small-cell environment, the signal-to-noise ratio at the receiver is expected to be high, and the quality of the link $\alpha$ is expected to be large. Hence, the CFR protocol is most suitable for cooperation in small-cell environments.

The CFR protocol with CSI-T and ideal link between the cooperating base stations achieves the best performance, among the CFR protocol setups. The presence of channel state information at the transmitter improves the bit error rate performance by $1 \mathrm{~dB}$ at high SNR. When the link between the base stations is nonideal, in the worst case $(\beta=$ $\alpha$ ), the performance degradation is negligible. It is $.5 \mathrm{~dB}$ on average at high SNR for the case of no CSI-T and nondiscernible for the case of when CSI-T is available at the base station.

In Figure 3, we compare the joint probability of outage performance of the CFR protocol to that of the FR 1 and FR 2 direct transmission schemes, for assumed spectral efficiencies $R=2$ and $R=4 \mathrm{bps} / \mathrm{Hz}$, respectively. The joint probability of outage metric indicates the effect of the channel and the interference on the reliability of the system. It can be observed from Figure 3 that, as in Figure 2, the CFR protocol outperforms the FR2 and FR1 direct transmissions at high SNR. For the case of $R=2 \mathrm{bps} / \mathrm{Hz}$, the CFR protocol with CSI-T achieves the best performance for all the SNR values, with comparable performance to the FR2 protocol at SNR below $5 \mathrm{~dB}$. The crossing point between the FR1 protocol and the CFR protocol with CSI-T occurs at $10 \mathrm{~dB}$ for $R=2 \mathrm{bps} / \mathrm{Hz}$. For a higher spectral efficiency, namely, $R=$ $4 \mathrm{bps} / \mathrm{Hz}$, the crossing point occurs at medium values of SNR, around $15 \mathrm{~dB}$. The effect of the presence of CSI-T and 


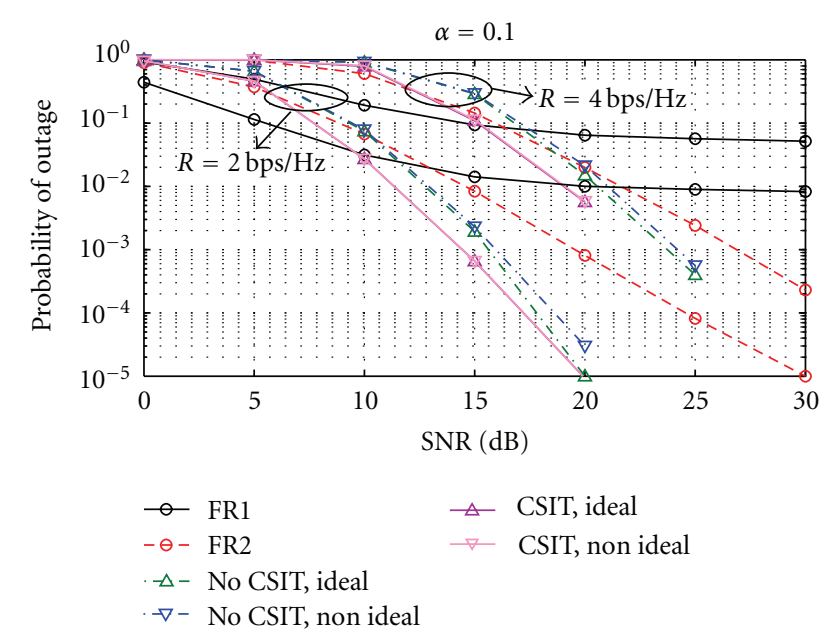

FIgURE 3: The joint probability of outage of $M_{0}$ and $M_{1}$, as a function of the SNR (dB), for two isolated base stations and two target spectral efficiencies $(R=2 \mathrm{bps} / \mathrm{Hz}$ and $R=4 \mathrm{bps} / \mathrm{Hz})$, for both the FR1 and the FR2 scenarios. The link quality is $\alpha=0.1$. The non ideal link denotes the case when the base station-to-base station link gain is $\beta=\alpha$.

the errors in the base station-to-base station links follow similarly the bit error rate performance in Figure 2. In particular, the presence of CSI-T incurs a gain of around $1 \mathrm{~dB}$ at high SNR, and the effect of the nonideality in the link is negligible, especially with CSI-T.

Figure 4 plots the average sum rate for various signal to noise ratio levels, when $\alpha=0.1$. It can be observed from the figure that the CFR protocol with CSI-T achieves the same average sum rate as the FR2 scheme with direct transmission, which is in accordance to the fact that diversity does not change the prelog factor in the capacity expressions. This average sum rate degrades when channel state information is not present at the transmitter. The effect of CSI-T is on average $1 \mathrm{~dB}$ increase in sum rate. The effect of the errors in the base station-to-base station link is again negligible in the presence of CSI-T at the base station.

The effect of $\alpha$, the quality of the adjacent base station link is important for small-cell scenarios, as it gives us an indication on how the CFR protocol would behave as the radius of the cell decreases and the base stations become closer to the mobile users they are serving. Figures 5, 6, and 7 plot, respectively, the bit error rate, the joint probability of outage and the average achieved sum rate as a function of signal-to-noise ratio, for $\alpha=0.25$. It can be observed from the figures that the CFR protocol performance improves as $\alpha$ increases. In particular, the crossing points between the FR1 direct transmission curves and those of the CFR protocol shift to the left by $5 \mathrm{~dB}$ for 16-QAM modulation and $2 \mathrm{~dB}$ for 4-QAM modulation in the bit error rate curves. For the sum-rate curves, the crossing point between the FR1 direct transmission and the CFR protocol is shifted by $8 \mathrm{~dB}$ to the left. As expected, as the value of $\alpha$ is increased, the value of the cooperation increases, and the CFR protocol performance becomes superior to direct transmission for most values of signal-to-noise ratio at the mobile station.

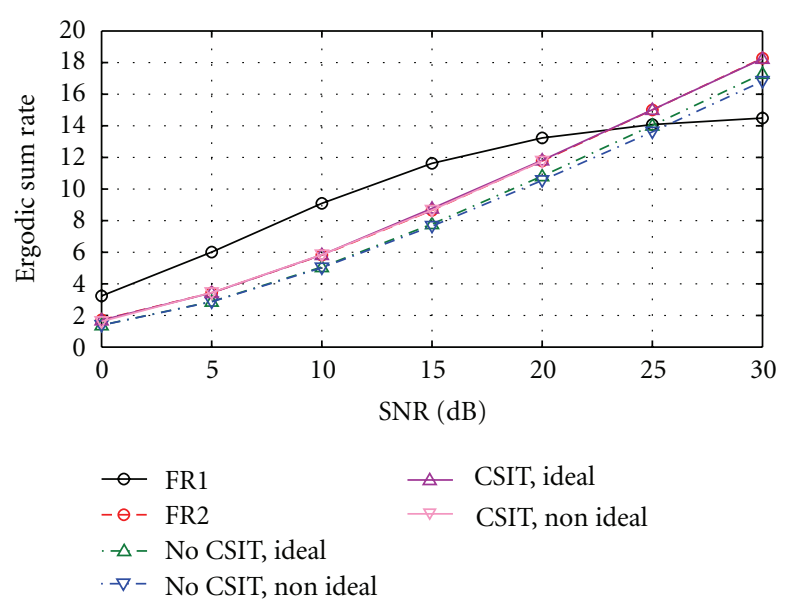

FIGURE 4: Average sum rate of $M_{0}$ and $M_{1}$, as a function of the SNR (dB), for two isolated base stations. The link quality is $\alpha=0.1$. The non ideal link denotes the case when the base station-to-base station link gain is $\beta=\alpha$.

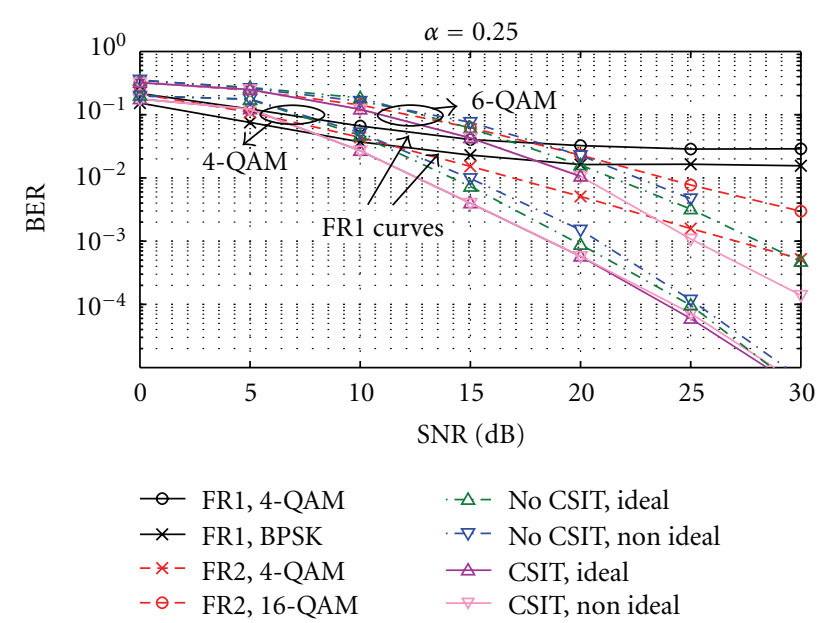

Figure 5: Bit error rate (BER), as a function of the SNR (dB), for two isolated base stations. The link quality is $\alpha=0.25$, and the modulation is 4-QAM and 16-QAM, respectively. The FR1 curves denote the direct transmission scheme with BPSK and 4-QAM modulation, respectively. The non ideal link denotes the case when the base station-to-base station link gain is $\beta=\alpha$.

For small-cell scenarios, where the received signal power at the mobile station is higher than that in a macro base station environment, because of the proximity of the base station to the mobile user, the CFR protocol is the winning strategy.

To further investigate the effect of $\alpha$ on the performance of the schemes investigated in this paper, we plot, in Figures 8 and 9, respectively, the bit error rate of the 4-QAM modulation scheme and the joint probability of outage for a target spectral efficiency $R=2 \mathrm{bps} / \mathrm{Hz}$, as a function of increasing $\alpha, 0<\alpha<1$, for a medium SNR value of $15 \mathrm{~dB}$. The direct transmission classical FR2 scheme is shown in the Figures for comparison as well as the FR1 direct transmission.

It can be observed from Figure 8 that the bit error rate of the CFR protocol exhibits a generally decreasing behavior 


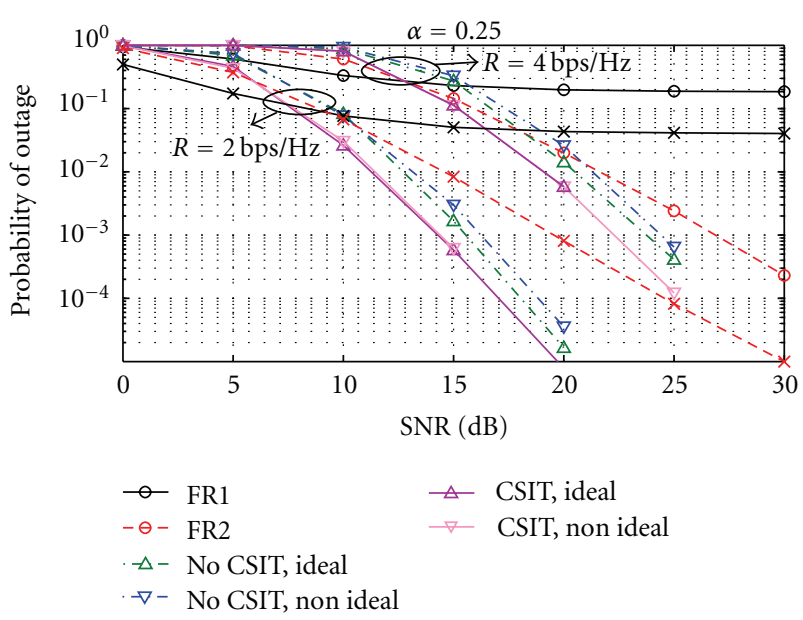

Figure 6: The joint probability of outage of $M_{0}$ and $M_{1}$, as a function of the SNR $(\mathrm{dB})$, for two isolated base stations and two target spectral efficiencies $(R=2 \mathrm{bps} / \mathrm{Hz}$ and $=4 \mathrm{bps} / \mathrm{Hz})$, for both the FR1 and the FR2 scenarios. The link quality is $\alpha=0.25$. The non ideal link denotes the case when the base station-to-base station link gain is $\beta=\alpha$.

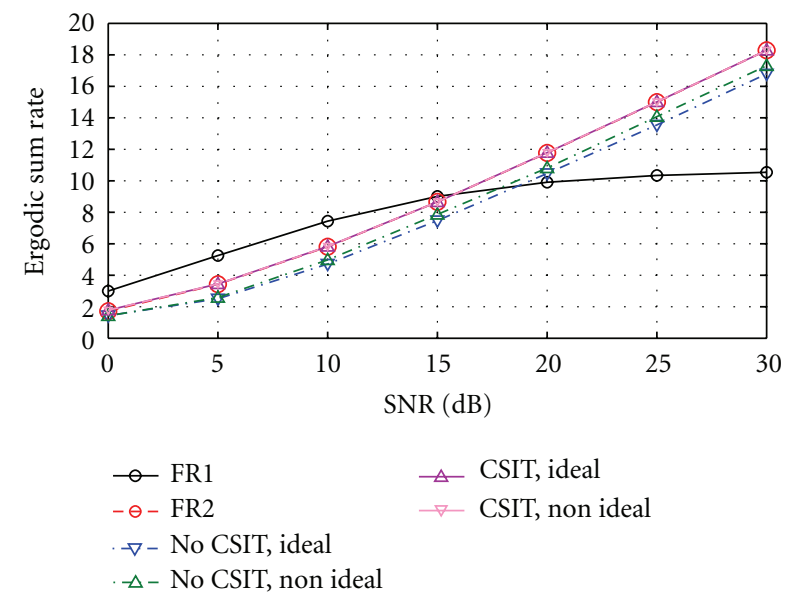

FIgURE 7: Average sum rate of $M_{0}$ and $M_{1}$, as a function of the SNR (dB), for two isolated base stations. The link quality is $\alpha=0.25$. The non ideal link denotes the case when the base station-to-base station link gain is $\beta=\alpha$.

as $\alpha$ increases. For $\alpha<0.5$, in the absence of CSI-T, the bit error rate remains unchanged as $\alpha$ increases; this can be explained by the fact that the collaboration link does not have the sufficient quality to increase the benefit. The bit error rate thus remains constant, but at a value that is lower than the classical FR2 scheme. For $\alpha>0.5$, the slope of decay of the bit error rate with $\alpha$ increases. The gap between the ideal and the nonideal worst case link between the base stations increases as $\alpha$ increases. This makes sense since we set the worst case link quality between the base stations to $\beta=\alpha$. In practice, the performance achieved by the CFR protocol with no CSI-T occurs between the two curves, as they correspond to two extreme cases of $\beta$. When statistical CSI-T is available at the base station, the effect of $\alpha$ is not as

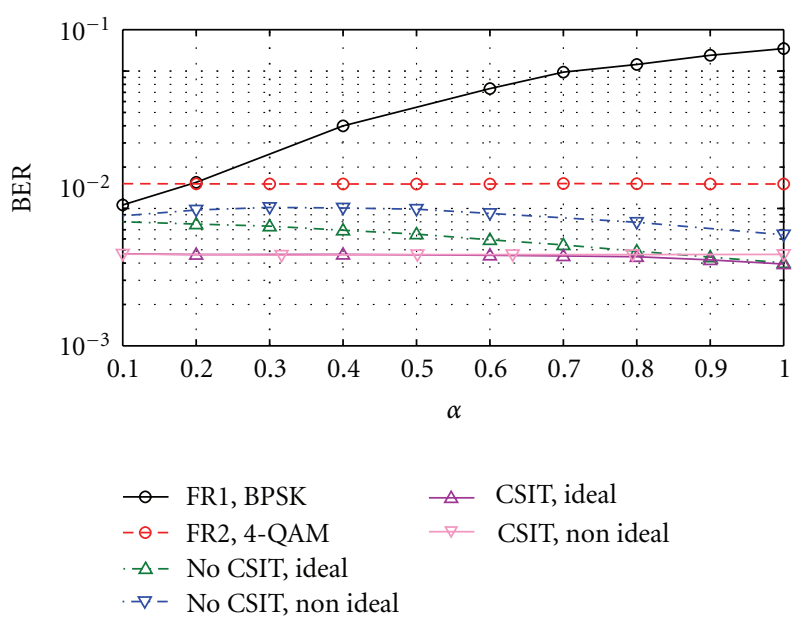

Figure 8: Bit error rate for 4-QAM symbols, as a function of $\alpha$, $0.1 \leq \alpha \leq 1$, for two isolated base stations and SNR $=15 \mathrm{~dB}$.

discernible as the case with no CSI-T. In fact, the bit error rate for CSI-T with nonideal link starts decreasing for very high values of $\alpha \approx 0$.9. Knowing the statistics of the channels at the transmitters shields the base stations against the effect of $\alpha$. The link quality has no effect on the bit error rate achieved by the FR2 classical transmission scheme as the base stations transmit on orthogonal frequency bands with no cooperation between base stations and no interference. The value of $\alpha$ has, however, an effect on the universal frequency reuse scheme FR1, where both base stations transmit at the same time on the same frequency band. As the value of $\alpha$ increases, the link quality between the adjacent base station and the mobile user improves, causing the cochannel interference from the adjacent base stations to increase and hence the probability of bit error to also increase.

The joint probability of outage, for $R=2 \mathrm{bps} / \mathrm{Hz}$, shown in Figure 9, exhibits the same behavior as that of the bit error rate curves. The FR1 direct transmission scheme is, however, always worse than the FR2 schemes and the CFR schemes, even for small values of $\alpha$. The joint probability of outage of the CFR protocol with CSI-T decreases with $\alpha$ starting at $\alpha=0.7$ for the ideal case, and $\alpha=0.8$ for the nonideal base station-to-base station link.

We finally examine the performance of the CFR protocol when the interference from the base stations outside the collaborating pair of interest is taken into account. We consider a linear array of $N=10$ base stations that form 5 collaborating pairs. Figures 10, 11, and 12 present, respectively, the bit error rate, the joint probability of outage and the achievable average sum rate, for the collaborating pair, given the interference from neighboring base stations. For the bit error-rate, the symbols are taken from a 4QAM constellation, and the target spectral efficiency for the probability of outage is set to $R=2 \mathrm{bps} / \mathrm{Hz}$. The link quality is set to $\alpha=0.1$. The figures compare the performance of the CFR protocol, without channel state information at the transmitter, for both an ideal link between the base stations and a worst case base station-to-base station link degradation. It can be observed from the figures that the 


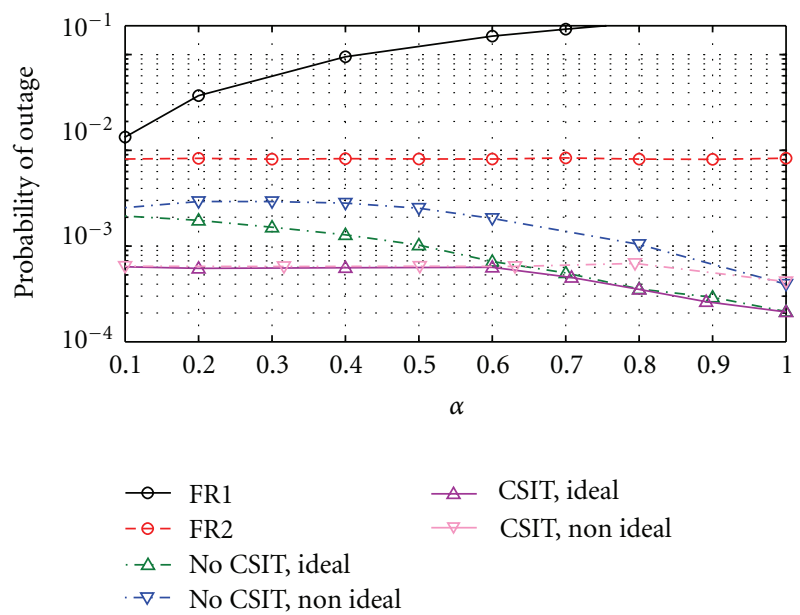

FIGURE 9: Joint probability of outage for a target spectral efficiency $R=2 \mathrm{bps} / \mathrm{Hz}$, as a function of $\alpha, 0.1 \leq \alpha \leq 1$, for two isolated base stations and $\mathrm{SNR}=15 \mathrm{~dB}$.

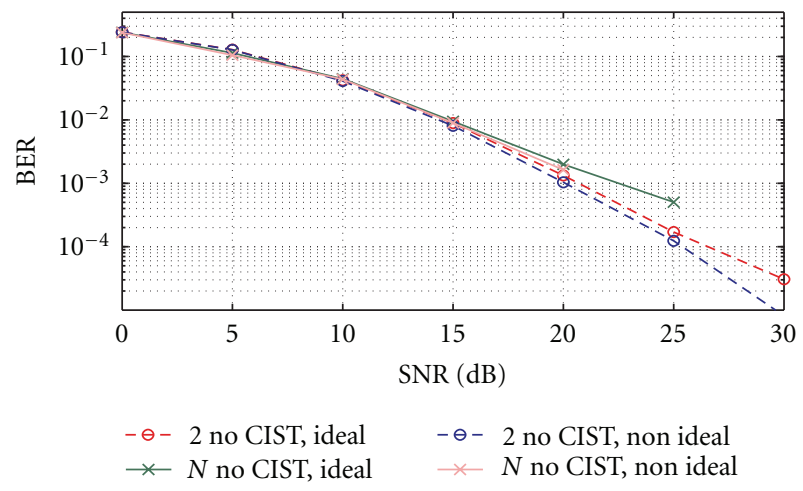

FIgURE 10: Bit error-rate for 4-QAM symbols, as a function of the SNR, for $N=10$ base stations with collaborating CFR pairs, and $N=2$ isolated BSs. $\alpha=0.1$.

effect of the interference from adjacent base stations is negligible and only discernible at high SNR; as the SNR increases, the reliability (bit error rate and joint probability of outage) as well as the average sum rate of the CFR protocol decreases with interference. The effect of the quality of the base station-to-base station link for the $N$ base stations is the same as that for the case of two isolated base stations. The performance of the CFR protocol decreases in the presence of error on the base station-to-base station link.

\section{Conclusion and Future Work}

In this paper, we proposed a novel distributed cooperation protocol called cooperative frequency reuse that is used on the downlink of frequency reuse small-cell systems. Collaboration between pairs of adjacent base stations enables the use of space as well as frequency diversity, at full rate, while keeping the half-duplex mode assumption. The protocol is based on a distributed space-time code derived from the Golden code. We discussed the performances of the protocol in terms of bit error rate, joint probability of

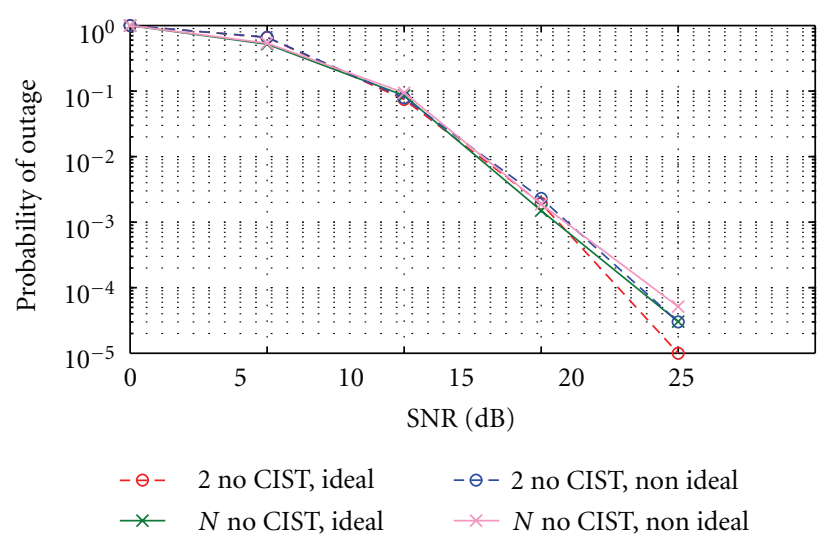

FIGURE 11: Joint probability of outage for a target spectral efficiency $R=2 \mathrm{bps} / \mathrm{Hz}$, as a function of the SNR, for $N=10$ base stations with 5 collaborating CFR pairs and $N=2$ isolated BSs. $\alpha=0.1$.

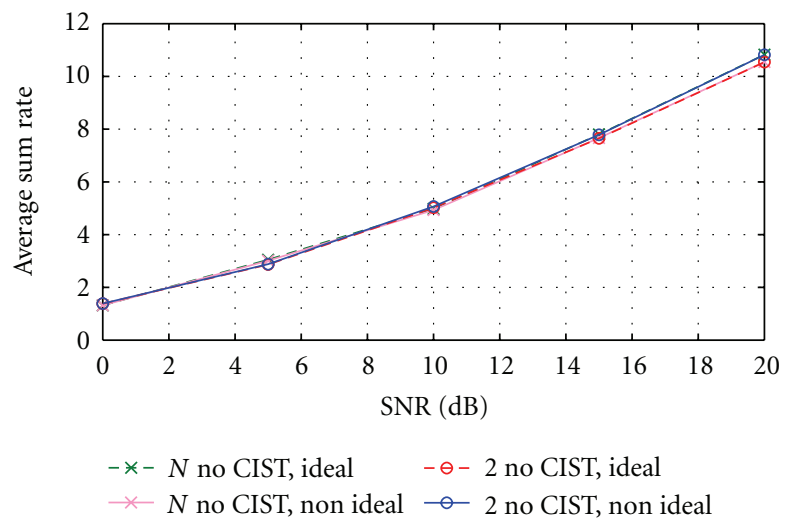

FIGURE 12: Average sum rate, as a function of the SNR, for $N=10$ base stations with 5 collaborating CFR paris and $N=2$ isolated BSs. $\alpha=0.1$ and the modulation scheme is 4-QAM.

outage, and ergodic sum rate under different scenarios. Simulation results showed that the CFR protocol increases the reliability of the cellular system, especially at high SNR, without incurring additional complexity overhead.

Future work includes an analytical analysis of power control optimization problems under partial (statistical) channel state information. It also includes accounting for fractional frequency reuse in the network model and optimizing the fractional part of the OFDM band under the CFR protocol. Finally, we will consider optimizing the duration of each phase within the frame to increase the reliability of the CFR protocol.

\section{Acknowledgments}

This work has been supported by the Agence Nationale de la Recherche within the project ANR-09-VERS0: ECOSCELLS. Robert W. Heath Jr. was supported by the National Science Foundation under Grant CNS-626797 and CCF-0830615. 


\section{References}

[1] M. S. Alouini and A. J. Goldsmith, "Area spectral efficiency of cellular mobile radio systems," IEEE Transactions on Vehicular Technology, vol. 48, no. 4, pp. 1047-1066, 1999.

[2] V. Chandrasekhar, J. G. Andrews, and A. Gatherer, "Femtocell networks: a survey," IEEE Communications Magazine, vol. 46, no. 9, pp. 59-67, 2008.

[3] S. Ramanath, M. Debbah, E. Altman, and V. Kumar, "Asymptotic analysis of precoded small cell networks," in Proceedings of IEEE INFOCOM, pp. 1-8, San Diego, Calif, USA, May 2010.

[4] F. Wang, A. Ghosh, C. Sankaran, P. J. Fleming, F. Hsieh, and S. J. Benes, "Mobile WiMAX systems: performance and evolution," IEEE Communications Magazine, vol. 46, no. 10, pp. 41-49, 2008.

[5] 3GPP TR 36.814, "Further advancements for E-UTRA physical layer aspects," 3rd Generation Partnership Project, Technical Specification Group Radio Access Network, February 2009.

[6] J. N. Laneman, D. N. C. Tse, and G. W. Wornell, "Cooperative diversity in wireless networks: efficient protocols and outage behavior," IEEE Transactions on Information Theory, vol. 50, no. 12, pp. 3062-3080, 2004.

[7] S. Shamai and B. M. Zaidel, "Enhancing the cellular downlink capacity via co-processing at the transmitting end," in Proceedings of the IEEE Vehicular Technology Conference (VTC '01), vol. 3, pp. 1745-1749, May 2001.

[8] J. Zhang, R. Chen, J. G. Andrews, A. Ghosh, and R. W. Heath, "Networked MIMO with clustered linear precoding," IEEE Transactions on Wireless Communications, vol. 8, no. 4, pp. 1910-1921, 2009.

[9] Y. Song et al., "Relay station shared by multiple base stations for inter-cell interference mitigation," IEEE C802.16m08/1436r1, November 2008.

[10] A. Sendonaris, E. Erkip, and B. Aazhang, "User cooperation diversity part I: system description," IEEE Transactions on Communications, vol. 51, no. 11, pp. 1927-1938, 2003.

[11] J. N. Laneman and G. W. Wornell, "Distributed space-timecoded protocols for exploiting cooperative diversity in wireless networks," IEEE Transactions on Information Theory, vol. 49, no. 10, pp. 2415-2425, 2003.

[12] M. Badr and J. C. Belfiore, "Distributed space time codes for the Amplify-and-Forward multiple-access relay channel," in Proceedings of the IEEE International Symposium on Information Theory (ISIT '08), pp. 2543-2547, July 2008.

[13] C. Hucher, G. R. B. Othman, and J. C. Belfiore, "AF and DF protocols based on Alamouti ST code," in Proceedings of the IEEE International Symposium on Information Theory (ISIT '07), pp. 1526-1530, June 2007.

[14] S. Jing, D. N. C. Tse, J. B. Soriaga, J. Hou, J. E. Smee, and R. Padovani, "Downlink macro-diversity in cellular networks," in Proceedings of the IEEE International Symposium on Information Theory (ISIT '07), pp. 1-5, June 2007.

[15] S. Shamai (Shitz), O. Somekh, and B. M. Zaidel, "Multicell communications: an information theoretic perspective," in Proceedings of the Joint Workshop on Communications and Coding (JWCC'04), October 2004.

[16] M. Debbah, "Mobile flexible networks: the challenges ahead," in Proceedings of the International Conference on Advanced Technologies for Communications (ATC '08), pp. 3-7, October 2008.

[17] S. W. Peters and R. W. Heath Jr., "The future of WiMAX: multihop relaying with IEEE $802.16 \mathrm{j}$," IEEE Communications Magazine, vol. 47, no. 1, pp. 104-111, 2009.
[18] M. Janani, A. Hedayat, T. E. Hunter, and A. Nosratinia, "Coded cooperation in wireless communications: space-time transmission and iterative decoding," IEEE Transactions on Signal Processing, vol. 52, no. 2, pp. 362-371, 2004.

[19] J. C. Belfiore, G. Rekaya, and E. Viterbo, "The golden code: a $2 \times 2$ full-rate space-time code with nonvanishing determinants," IEEE Transactions on Information Theory, vol. 51, no. 4, pp. 1432-1436, 2005.

[20] S. Yang and J. C. Belfiore, "Optimal space-time codes for the MIMO amplify-and-forward cooperative channel," IEEE Transactions on Information Theory, vol. 53, no. 2, pp. 647663, 2007.

[21] A. D. Wyner, "Shannon-theoretic approach to a Gaussian cellular multiple-access channel," IEEE Transactions on Information Theory, vol. 40, no. 6, pp. 1713-1727, 1994.

[22] S. Jing, D. N. C. Tse, J. B. Soriaga, J. Hou, J. E. Smee, and R. Padovani, "Multicell downlink capacity with coordinated processing," EURASIP Journal on Wireless Communications and Networking, vol. 2008, Article ID 586878, 2008.

[23] O. Somekh, B. M. Zaidel, and S. Shamai, "Sum rate characterization of joint multiple cell-site processing," IEEE Transactions on Information Theory, vol. 53, no. 12, pp. 4473-4497, 2007.

[24] O. Simeone, O. Somekh, H. V. Poor, and S. Shamai, "Local base station cooperation via finite-capacity links for the uplink of linear cellular networks," IEEE Transactions on Information Theory, vol. 55, no. 1, pp. 190-204, 2009.

[25] S. Shamai and A. D. Wyner, "Information-theoretic considerations for symmetric, cellular, multiple-access fading channels - Part I," IEEE Transactions on Information Theory, vol. 43, no. 6, pp. 1877-1894, 1997.

[26] O. Somekh and S. Shamai, "Shannon-theoretic approach to a Gaussian cellular multiple-access channel with fading," IEEE Transactions on Information Theory, vol. 46, no. 4, pp. 1401$1425,2000$. 